\title{
COEXISTENCE OF ANTI-NEUREXIN 3 AUTOIMMUNE ENCEPHALITIS WITH SYSTEMIC LUPUS AND SECONDARY SJÖGREN'S SYNDROME
}

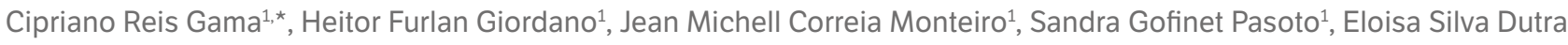 \\ de Oliveira Bonfá ${ }^{1}$
}

1.Universidade de São Paulo, São Paulo (SP), Brazil.

*Corresponding author: ciprianogama@gmail.com

\section{BACKGROUND}

The coexistence of autoimmune encephalitis (AE) with autoimmune diseases is rare. Its autoantibody characterization and possible distinct clinical expression remain undetermined. The purpose of this report is to describe the unusual association of AE due to anti-neurexin 3 expression with two autoimmune conditions: systemic lupus erythematosus (SLE) and Sjögren's syndrome (SS).

\section{CASE REPORT}

Female patient, 43 years old, had new onset of migratory headache associated with polyarthralgia and reported yellow fever vaccination six months after the onset of symptoms. In three days, she evolved to a worsening headache, associated with prostration and generalized tonic-clonic seizures. Orotracheal intubation was performed and, during sedation, she had several partial epileptic seizures which hampered the reduction of sedatives and evolved with neck stiffness. Cerebrospinal fluid showed a total of 15 cells ( $94 \%$ of lymphocytes), protein $37 \mathrm{mg} / \mathrm{dL}$, glucose $81 \mathrm{mg} / \mathrm{dL}$, fungus, bacilloscopy and cultures negative, polymerase chain reaction negative for yellow fever, dengue fever, CMV, EBV, chickenpox and herpes simplex I and II. Serology studies were negative for yellow fever, dengue fever, Rocio and Saint Louis encephalitis, HIV I/II, syphilis, HTLV I/II and hepatitis B and C. Brain CT and MRI were unremarkable. Empirical treatment was started with ceftriaxone and acyclovir. Laboratory evaluation evidenced homogeneous nuclear pattern (1/1280), positive anti-ds-DNA (106 U/mL, Crithidia positive), positive anti-SSA (>200 U/mL), hypocomplementemia (C3: 72 and C4: 4.9). Anti-Sm, SS-B, ANCA and antiphospholipids were negative. Complementary investigation for AE identified the presence of anti-neurexin $3 a$. Diagnosis of lupus was confirmed and methylprednisolone $1 \mathrm{~g}$ per day $/ 5$ days was started concomitant with intravenous human immunoglobulin (total dose: $2 \mathrm{~g} / \mathrm{kg}$ ), followed by prednisone $60 \mathrm{mg} /$ day and azathioprine with clinical improvement. After one week, she presented with persecutory delusion, agitation and auditory hallucinations treated with haloperidol. She did not relapse neurological symptoms and remained asymptomatic for other lupus manifestations. A new onset of polyclonal hypergammaglobulinemia, sicca manifestations, confirmed by positive Schirmer test, and a minor salivary gland biopsy with focus score of 1.1 occurred within three years of follow-up. Therefore, SS association was confirmed with SLE and AE.

\section{CONCLUSION}

This rare case of AE with concomitant two autoimmune disease associated with anti-neurexin-3 antibodies revealed a pattern of severe disease but responsive to treatment. Immunosuppressive therapy and IVIg was effective in counterbalancing the reported effect of reduced neurexin-3 and alteration of synapse development induced by the antibody.

\section{KEYWORDS}

Autoimmune encephalitis, Systemic lupus erythematosus, Secondary Sjögren's syndrome, Anti-neurexin 3 autoimmune encephalitis. 